\title{
Research on BPS of Railway Prestressed Concrete Beam Intelligent Tension System
}

\author{
Li Haopeng \\ Jilin Railway Technology College, Jilin, China \\ 24702296@sina.com
}

Keywords: BPS, Railway Prestressed Concrete Beam, Intelligent Tension System

\begin{abstract}
In the process of large beam production, its tension technology is a relatively important link. In the process of tensioning, due to the direct impact of its prestress loss, the accuracy and friction of the tensioning system, and the situation of the staff, it is difficult to guarantee the quality of the precast concrete beam. If the tension of the prestressing tension is difficult to control, the lighter condition will cause some cracks in the anchoring end of the beam. In severe cases, the cracks and the prestressing tendons will be torn off, which will eventually cause the prestressed structure to be damaged. . Therefore, in the study of high-intelligence, full-process, high-precision precast concrete beam pre-automatic system, not only can the effectiveness of the test data be guaranteed, but also the construction safety of prestress can be guaranteed.
\end{abstract}

\section{Introduction}

With the rapid development of the economy, there are more and more problems in the pre-stress intelligent tension control process. The main goal of the research is how to improve the tension control precision of the pre-stress, and control the pre-control from all sides. The magnitude of the stress and the specific conditions of the dispersion ensure the tension of the bridge prestress during the construction process, and the specific requirements of each aspect are in accordance with the design specifications. Contemporary, domestic and international research on tension technology is mainly divided into two different types of ideas, one is digital oil pump technology, and the other is prestressed construction information.

\section{Construction technology of prestressed concrete beam prestressed intelligent tensioning system in railway engineering}

The intelligent tensioning system is mainly composed of four parts, mainly including sensor system, auxiliary system, intelligent control system, mechanical power system and so on. This system adopts a high-degree pressure sensor to measure directly on the tensile force, adopts the measured elongation value of the digital displacement sensor, and uses a high-performance hydraulic system as its power loading system, using a professional programming controller. The control system can be managed, and a computer management system can be used to effectively manage the tension data.

During the construction process: first install the anchor, then install the sensor, then install the connecting plate, and finally start the control system. In the tensioning process, we need to use the tension control as the main index, and the prestressing tendon elongation is proofread. In the process of passing the sensor through the system, the sensor is used to effectively collect the tension value of each jack. And scientific judgment of each data, so that it can accurately grasp its tension and speed loading. When the system is in accordance with various technical requirements, the pre-stress tensioning process is automated. At the same time, it also realizes the functions of analyzing charts, querying historical data, and transferring stored data.

Set the parameters. We can input different types of models, tensile strength values, elongation check values, numbering, etc., and also use wireless communication technology to set different 
parameters for the tensioning technology of different types of bridges. Calibration tension. The tensile force of the system can be detected and calibrated by a standard test ring or a force measuring machine. Tension synchronization. We can automatically control the tension in one click according to the process parameters to be entered. In the process of tensioning, we can automatically control the two ends of the prestress to maintain the same elongation, so that the tension can be adjusted and the tension synchronization function can be better realized. Automatically record measurement data. The elongation value of the strand and the specific val Store valid data. The whole process data can realize automatic data acquisition, storage of information, drawing tension, drawing elongation curve, etc. After the process is finished, the calculation result can be automatically analyzed and the data can be stored effectively. Transfer data. The display of the chart data can be systematically displayed by wireless transmission or transmission of a moving medium. In the process of historical data query and curve drawing, network data can be transmitted when exporting data.

\section{BPS intelligent tension system design}

The BPS intelligent tensioning system consists of intelligent tensioning hardware devices and software platforms. The hardware equipment consists of four parts: a smart main pump station, a smart auxiliary pump station, a smart jack, and a smart handheld radio frequency identification terminal. The software platform includes SPT software for use on the intelligent pumping station, Station client software running on the field operation center, Cloud server software running on the cloud server, Client client software running on the PC platform, and running on the personal mobile phone. Portable client software on the platform. The composition of the station. The hardware equipment consists of four parts: a smart main pump station, a smart auxiliary pump station, a smart jack, and a smart handheld radio frequency identification terminal. The software platform includes SPT software for use on the intelligent pumping station, Station client software running on the field operation center, Cloud server software running on the cloud server, Client client software running on the PC platform, and running on the personal mobile phone. Portable client software on the platform.

The intelligent pumping station is the power source of the tensioning system, including the main pump and the auxiliary pump. The hydraulic power output from the pumping station drives the jack to extend the cylinder and reduce the cylinder. The intelligent pumping station is composed of industrial tablet computers, fuel tanks, motors, hydraulic pumps, hydraulic valve sets, piping systems, and electric control boxes. The intelligent control part of the intelligent pumping station is composed of two parts: the control box and the control panel. The control box is composed of a PLC microcomputer control unit, an analog measurement and detection unit (including displacement and pressure detection), a power protection unit, a motor control unit, a communication unit, and a temperature and humidity control unit. The control panel consists of an industrial tablet and control buttons that complete the human interface function.

The intelligent pumping station can accurately realize the commands set by the program, and ensure the reliable interaction of data communication through the Ethernet wireless communication interface. Each station can work online or independently. In online mode, all control commands are issued by the master station, and the pump station integrates the mobile $4 \mathrm{G}$ data module to upload the results to the server. The intelligent jack is mainly composed of a $350 \mathrm{t}$ jack and a piston extension measuring device, a clip exposure measuring device and a connecting cable, and transmits the real-time displacement data of the tensioning process to the control system of the intelligent pumping station through the data line for real-time operation. monitor. The $350 \mathrm{t}$ jack is mainly composed of three parts. The first is the "unmovable body" composed of the oil cylinder, the piercing sleeve, the positioning nut, the large plug, the rear sealing plate, the rear compression ring and its seal. And the "sports body" composed of its seals; the third is the handle part that is easy to lift. 


\section{BPS intelligent tension software platform development}

The BPS intelligent tension software platform develops different application software for the needs of on-site construction workers, management personnel and owner supervisors. The hardware environment according to the program operation is divided into: 1 SPT software running on the intelligent pump station; 2 running in BPS Station software on the beam farm server; 3 BPS Cloud software running on the cloud server; 4 BPS Client software running on the remote client. 5 BPS Portable software running on the handheld terminal.

(1) The automated BPS system is based on automatic tensioning equipment, with automatic tension control of pre-stressed beam prestressing construction, automatic measurement of tension control indicators, automatic preparation of tension data reports, etc., to achieve automation of prestressed tension control process. (2) The refinement automatic tension control device monitors the oil pump in real time through the precise sensing system feedback, which can control the tension deviation range to about $1 \%$, and realize multi-top simultaneous operation to improve the tension synchronization. In addition, the high-precision displacement sensor is used to measure the tensile elongation of the steel beam, so as to achieve precise control and accurate measurement during the tensioning process. (3) Informatization BPS system can use RFID technology to track the identity information, beam type information, progress information and quality information of the beam from construction to operation, and realize the informationization of the bridge life cycle. (4) The BPS system equipped with video surveillance equipment can obtain the video information of the construction site through the functions of pan/tilt operation and video playback of the system software. The software can also query the prestressed tension process through various forms such as graphs and tables. The data results to visualize the precast beam construction process. (5) The remote BPS system relies on "cloud technology". It can view the data information, construction progress information and live video information in the place where there is Internet, and realize the remote control of construction supervision and management.

\section{BPS advantage}

For the mechanical power system, it is necessary to stabilize its performance, and at the same time, it must be easy to use and can be used normally under high temperature conditions or severe cold conditions. Contemporary, oil pressure control work is mainly realized by electro-hydraulic servo valve, but electro-hydraulic servo valve has higher requirements on precision, and has strict requirements on the clean condition of the oil passage and the quality of the valve body.

The development of scientific and reasonable sensing equipment requires simple installation, high precision, good linearity, good weather resistance and strong anti-interference ability. According to the various aspects of the system structure and the requirements of various technical conditions, the wire-type displacement sensing line can be applied by performing indoor and outdoor verification and performance selection for various sensors. Generally speaking, unbonded prestressing can be used to perform construction work after the vertical line.

For intelligent tensioning systems, the core is subsystem control. Based on the programming controller, we are in the process of programming the pre-stressed concrete bridge hardware and software control system. The controller is mainly used in an automatic control system with a poor objective environment and high reliability and safety. The work of the control subsystem is divided into two aspects, mainly including software design and hardware design. The system software design mainly includes three parts: main program, subroutine and interrupt program. The system hardware includes data acquisition module, electrical design and design anti-interference measures, and PLC controller. This system has many advantages, such as: perfect function, strong performance, strong adaptability and so on.

For the intelligent tensioning system, it is necessary to configure an auxiliary system with higher safety and more complete functions, so as to ensure the personal safety and bridge quality of the staff. The specific functions are as follows: (1) In the event of an abnormal power outage, the current data can be automatically saved, and the breakpoint position can continue to resume its 
power-off. (2) In the hydraulic system, it can stabilize its intelligent oil control system. (3) Automatically monitor the jacks from all sides to further avoid the phenomenon that the jacks are topped up due to excessive oil pressure. (4) Ensuring that its detection software is in use and capable of intelligent diagnosis. (5) When the data times out or an abnormal soil occurs, the alarm is automatically performed. (6) With remote control transmission function and wireless data transmission function.

(1) Requires its intelligent tensioning equipment to have high precision. In the course of work, any small mistakes or minor faults may cause the tension data to exceed the standard. Therefore, it is necessary to systematically train their staff so that they can master various operational skills and ensure smooth and orderly work during the operation. And continue to strengthen the maintenance and maintenance of the equipment, if any problems occur, they need to be resolved in a timely manner. Accurate application of pre-stress is a critical task in ensuring the safety of the bridge structure. (2) During the process of installing the anchor head and the prestressed pipeline, it is necessary to carefully review the coordinates of the joints to ensure that all work is carried out according to the construction requirements, and the loss of prestress caused by the friction of the pipeline is reduced from all sides.

\section{Conclusion}

Prestressed tension construction is the key link in the production process of railway prestressed concrete precast beam. The traditional manual operation method has the disadvantages of low precision, low efficiency, complicated operation, poor synchronization, high calibration frequency and uncontrollable result authenticity. In view of the existing problems of traditional prestressed artificial tension of railway bridges, the article is based on big data, cloud technology and radio frequency identification technology. It has developed a set of intelligent tension pumping station, intelligent tensioning jack equipment, intelligent software platform and intelligentization. Handheld equipment integrated railway prestressed concrete beam intelligent tension control and management system (BPS), and carried out test verification and application promotion in the Shiji passenger line.

\section{References}

[1] WU Chong, XIAO Xianglin. Research on Prestressed Automatic Tensioning System of Railway Prefabricated Beam [J]. Railway Engineering, 2014, 54(11): 11 - 13.

[2] H De Backer, W De Corte, P Van Bogaert. A Case Study on Strain Gauge Measurements on Large Post-tensioned Concrete Beams of a Railway Support Structure [J]. Insight: Non Destructive Testing and Condition Monitoring, 2003, 45(12): 822 - 826.

[3] GUO Quanquan, LI Zhu, DUAN Lian. Automatic Post - Tensioningin Prestressed Concrete Structures [J]. Structural Engineering International: Journal of the International Association for Bridge and Structural Engineering (IABSE), 2004, 14(1): 37 - 41.

[4] LIAO Qiang, XU Jianmin, LI Wenfeng. Application of Presstressed Intelligent Tension System in Construction of Bridges [J] . Technologyof Highway and Transport, 2015, 32(2): 102 - 105.

[5] LI Xiaobing, TIAN Shiqiang, CHEN Huaxiang. Application of Prestressed Tension Monitoring Equipment in Bridge Construction [J] . Technology of Highway and Transport, 2012, 8 (1): 139 141. 